\title{
Some Nano Bio-Mofs Evaluated For Storage Purpose of Drugs
}

\author{
Tabinda Sattar and Muhammad Athar* \\ Assistant professor, Institute of Chemical Sciences, Pakistan
}

Received: January 08, 2018; Published: January 25, 2018

*Corresponding author: Muhammad Athar, assistant professor, institute of chemical sciences, Bahaudin zakariya university Multan, Pakistan, Tel: 03234548374; Email:athar.qr@gmail.com

Abstract

Nano bio-MOF compounds 3-5 (copper serinate, copper prolinate and copper threoninate) have been evaluated for successful in vitro drugs adsorption of three drugs, named terazosine, telmisartan and glimpiride. Pore sizes and BET surface areas were recorded on N2 adsorption isotherms. In order to elaborate the phenomenon of in vitro drugs adsorption in all these compounds we obtained TGA and PXRD spectra of them. The high performance liquid chromatography (HPLC) was used to monitor the amounts of adsorbed drugs and its slow release from all these compounds.

Keywords: Nano bio-MOFs; Invitro; Drugs adsorption; TGA; PXRD; HPLC

Abbreviations: MOFs: Metal Organic Frameworks; SEM: Scanning Electron Microscopic; HPLC: High Performance Liquid Chromatography; TGA: Thermo Gravimetric Analysis

\section{Introduction}

Metal Organic Frameworks (MOFs) are the materials which are significant due to different applications especially in the field of drugs storage [1,2]. Still there is need of designing more compatible drugs carriers for successful drugs delivery applications both for in vitro and in vivo purposes. In these respect bio-metal organic frameworks (Bio-MOFs) is a useful extension of MOFs [3]. Actually bio molecule linkers and bio compatible metal cations can be utilized for the construction of bio-MOFs. These materials have great potential utility as targeted drugs carrier materials inside human body containing no harmful effects at all for health [4]. The MOF materials which were previously used for drugs carrying purposes faced some compatibility problems inside human body due to their large sizes [5]. So with the passage of time use of nano sized MOF materials became more feasible for drugs storage and release. Kitagawa et al. have reported the use of some large sized MOF materials used successfully for drugs storage purposes [6].

Latter on Rieter et al. have synthesized some nano scale MOFs and used them for drugs carrying, so having much better results as compared to previously reported drugs carrier MOFs. Wottki et al. [7] have reported some MOFs materials which were designed and tuned accordingly for usage as more potential nano carrier materials as compared to previously used drugs carrier materials $[8,9]$. Roder et al. [10] have also reported the successful use of nano sized MOF materials for drugs delivery. The smaller size and high surface areas these nano sized MOF materials make them the targeted drugs delivery vehicles [10]. The bio MOFs can be scaled down to the nano-regime as well in order to form nanoscale bio metal-organic frameworks (nano bio-MOFs). Nano bio MOFs are the nano sized MOF materials containing bio-molecules and biocompatible metal cations. This emerging class of nano-bio-MOFs can be considered as promising candidates for the drug adsorption and its controlled release due to their large surface areas, high porosity, and presence of bio compatible linker molecules [11]. Several research reports reveal the use of nano sized MOFs in the field of drugs delivery [12-14].

Three hydrothermally synthesized nano bio-MOFs compounds 3-5 (copper serinate, copper prolinate and copper threoninate) have been reported by Sattar et al. [15]. These compounds have been hydrothermally synthesized and characterized by Scanning Electron Microscopic (SEM) studies. Previously these materials were used as drug carrying for rosuvastatin drug. These materials were found feasible for drug storage with its slow release from the materials after intervals of time as monitored through High Performance Liquid Chromatography (HPLC). The present work is designed to elaborate the pore sizes and BET surface area calculations of these materials and also to evaluate their drug storage potential for three more drugs like terazosine hydrochloride, telmisartan and glimpiride. In vitro drugs adsorption studies of these drugs and their release may lead to the in vivo usage of these drugs delivery inside human body as these drugs have applications for hypertention and 
control blood sugar levels. Thermo gravimetric analysis (TGA) and powder X-rays Diffraction Analysis (Powder XRD) patterns of all these new compounds in pure form and after drugs adsorption have been recorded. The amounts of adsorbed drugs and its slow release after intervals have been monitored through the High Performance Liquid Chromatography (HPLC).

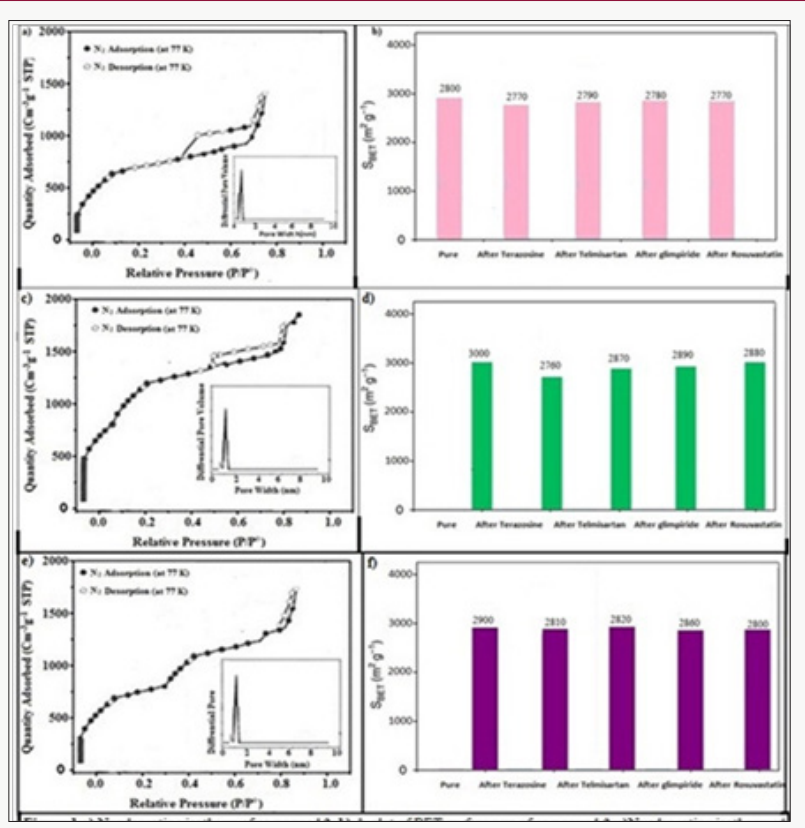

Figure 1: a) N2 adsorption isotherm of compound 3, b) A plot of BET surface area of compound 3, c) N2 adsorption isotherm of compound 4,d) A plot of BET surface area of compound 4, e) N2 adsorption isotherm of compound 5, f) A plot of BET surface area of compound 5 .

BET specific surface areas of compounds 3,4 and 5 have been determined as $2800 \mathrm{~m}^{2} \mathrm{~g}^{-1}, 2000 \mathrm{~m}^{2} \mathrm{~g}^{-1}$ and $2900 \mathrm{~m}^{2} \mathrm{~g}^{-1}$ respectively with a pore size of $10 \AA$ by using BET-method based calculations on $\mathrm{N}_{2}$ adsorption isotherm data. The Figure 1 represents the $\mathrm{N}^{2}$ adsorption isotherm of compound 3, 4 and 5. Four types of drugs (terazosine hydrochloride, telmisartan, glimpiride and rosuvastatin) have been absorbed into compounds 3,4 and 5 . It was also elaborated that some pores were blocked after the drugs adsorptions thus decreasing the specific surface areas of this material as shown in Figure 1. TGA plots of the as synthesized and drugs adsorbed compounds 3-5 were carried out on a SDT Q600 by heating the compounds from $0 \mathrm{oC}$ to $600 \mathrm{oC}$ at a heating rate of $10 \mathrm{oC}$ per minute. TGA plot (Figure 2a) before drugs adsorption shows first weight loss of $10 \%$ at $120 \mathrm{oC}$ which is due to the removal of water molecule. Up to $340 \mathrm{oC}$ the framework shows stability with no weight loss. Second weight loss is observed at $340 \mathrm{oC}$ which continues up to $400 \mathrm{oC}$ with a $40 \%$ weight loss due to the framework decomposition along with ligands. Then framework continues to decompose gradually until the whole framework decomposes till 590 oC with the formation of metal oxides (Figure 2).

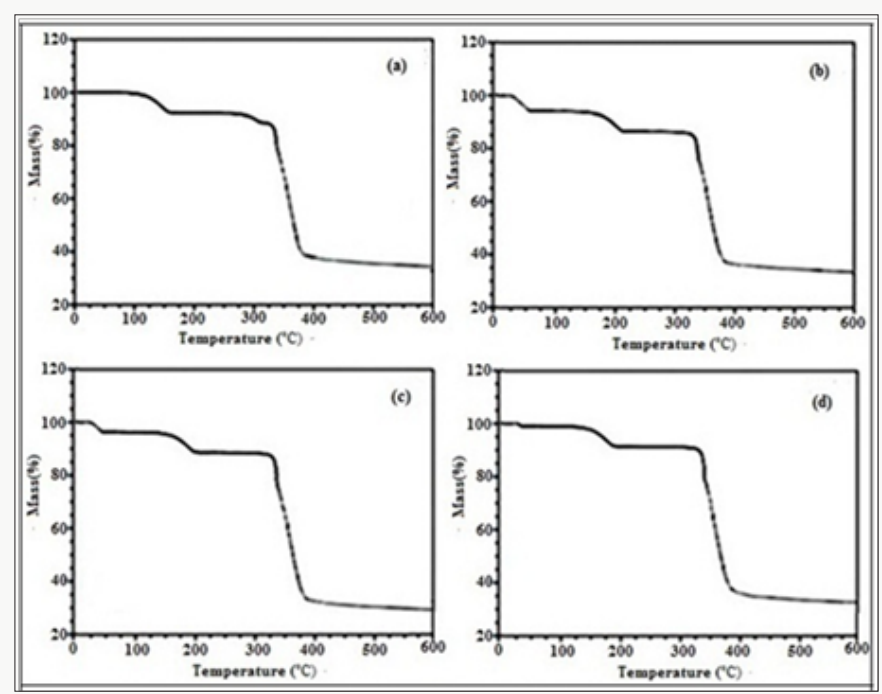

Figure 2: TGA plot of compound 3 a) before drugs loading, b) after terazosine hydrochloride loading, c) after telmisartan loading, d) after glimpiride loading. 
After the terazosine adsorption the TGA plot of compound 3 (Figure $2 \mathrm{~b}$ ) shows a weight loss of $9 \%$ at $40 \mathrm{oC}$, which may be attributed towards the loss of drug molecules. After that the framework remains intact for some time and then at $140 \mathrm{oC}$ the framework shows stability up to $180 \mathrm{oC}$. Second weight loss of $4 \%$ at $180 \mathrm{oC}$ is observed due to the loss of water molecule. After that at $280 \mathrm{oC}$ the framework starts to decompose up to $380 \mathrm{oC}$ with a weight loss of $58 \%$. No further weight loss observed for this material. TGA plot of telmisartan adsorbed compound 3 (Figure 2c) shows a weight loss of $4 \%$ at $40^{\circ} \mathrm{C}$ which is due to the loss of telmisartan contents. Another weight loss of $4 \%$ at $150^{\circ} \mathrm{C}$ shows the loss of water molecule. Then the framework starts to decompose at $250^{\circ} \mathrm{C}$ which continues till $550^{\circ} \mathrm{C}$ with a maximum weight loss of $59 \%$. Similarly TGA plot of glimpiride adsorbed compound 3 (Figure 3) shows first weight loss of $4 \%$ at $50^{\circ} \mathrm{C}$ which is due to the removal of glimpiride contents. At $170^{\circ} \mathrm{C}$ the framework shows a weight loss of $4 \%$ up to $190^{\circ} \mathrm{C}$ which is due to removal of water molecule. At $280^{\circ} \mathrm{C}$ the decomposition of the framework and ligands starts which continues gradually up till $560^{\circ} \mathrm{C}$ with maximum weight loss of $58 \%$.

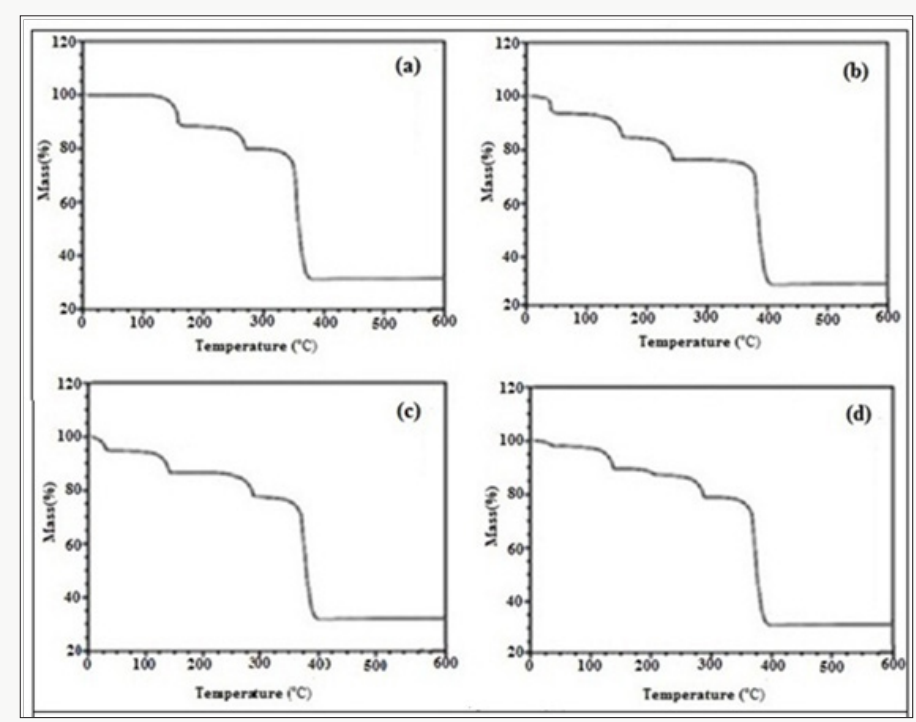

Figure 3: TGA plot of compound 4 a) Pure, b) after terazosine loading, c)after telmisartan loading, d) after glimpiride loading.

Figure 3a shows the TGA plot of compound 4, before drugs adsorption shows first weight loss of $4 \%$ at $180 \mathrm{oC}$ which is due to the removal of water molecules. Second weight loss is observed at $280 \mathrm{oC}$ which continues up to $380 \mathrm{oC}$ due to the start of framework decomposition along with ligands. Then framework continues to decompose gradually until the whole framework decomposes till $580 \mathrm{oC}$ with a maximum weight loss of $49 \%$ along with the formation of metal oxides. The TGA plot of compound 4 after the terazosine adsorption shows (Figure $3 \mathrm{~b}$ ) a weight loss of $5 \%$ at $60 \mathrm{oC}$, which is due to the loss of drug molecules. Then the framework shows stability up to $180 \mathrm{oC}$. Second weight loss of $9 \%$ at $180 \mathrm{oC}$ is due to the loss of coordinated water molecule. The framework remains intact till $280 \mathrm{oC}$, after that it starts to decompose and this decomposition continues up to $380 \mathrm{oC}$ with a weight loss of $58 \%$. Similarly TGA plot of telmisartan adsorbed compound 4 (Figure 3c) shows a weight loss of $4 \%$ at $40^{\circ} \mathrm{C}$ which is due to the loss of telmisartan contents. Another weight loss of $8 \%$ at $150^{\circ} \mathrm{C}$ shows the loss of water molecules. At $250^{\circ} \mathrm{C}$ the gradual decomposition of the framework starts which continues till $300^{\circ} \mathrm{C}$ with a maximum weight loss of $59 \%$.

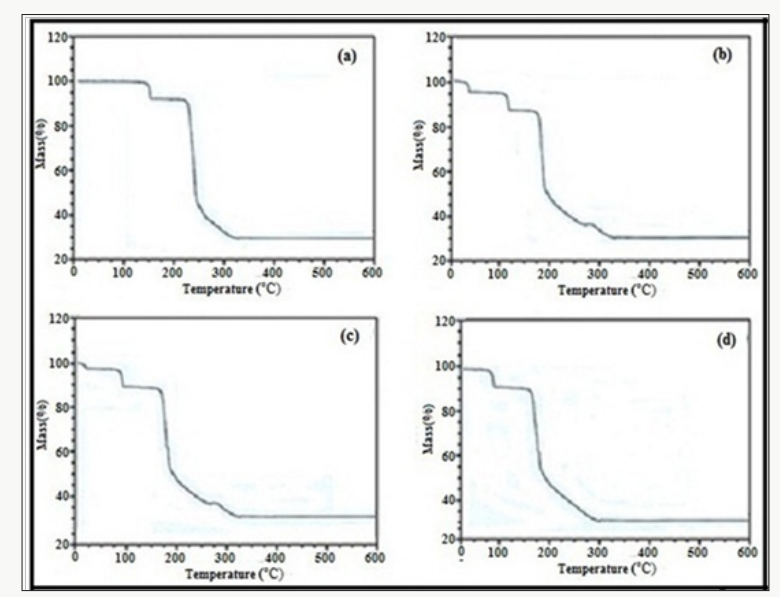

Figure 4: TGA plot of compound 5 a) Before drugs adsorption, b) after terazosine loading, c) after telmisartan loading, d) after glimpiride loading. 
A TGA plot of glimpiride adsorbed compound 4 (Figure 3d) shows first weight loss of $4 \%$ at $50^{\circ} \mathrm{C}$ which is due to the removal of glimpiride contents. At $170^{\circ} \mathrm{C}$ framework shows a weight loss of $8 \%$ up to $190^{\circ} \mathrm{C}$ which is due to removal of water molecule. At $280^{\circ} \mathrm{C}$ the decomposition of the framework and ligands starts which continues till $330^{\circ} \mathrm{C}$ with weight loss of $58 \%$ (Figure 3). A plot of rosuvastatin adsorbed compound 4 (Figure $3 \mathrm{e}$ ) shows first weight loss of $3 \%$ at $45^{\circ} \mathrm{C}$ which is due to the removal of drug contents. At $171^{\circ} \mathrm{C}$ the framework shows a weight loss of $8 \%$ which is due to the removal of water molecules. At $285^{\circ} \mathrm{C}$ the decomposition of the framework and ligands starts which continues gradually up to $560^{\circ} \mathrm{C}$ with a weight loss of $58 \%$. No further weight loss observed in the remaining range studied. TGA plot of compound 5 before drugs adsorption (Figure 4a) shows first weight loss of $7 \%$ at $1180 \mathrm{C}$ which is due to the loss of water molecule. At $300 \mathrm{oC}$ second weight loss is observed which continues up to $380 \mathrm{oC}$ with the start of framework decomposition along with ligands. Then framework continues to decompose gradually until the whole framework decomposes till 550 oCwith $50 \%$ weight loss.

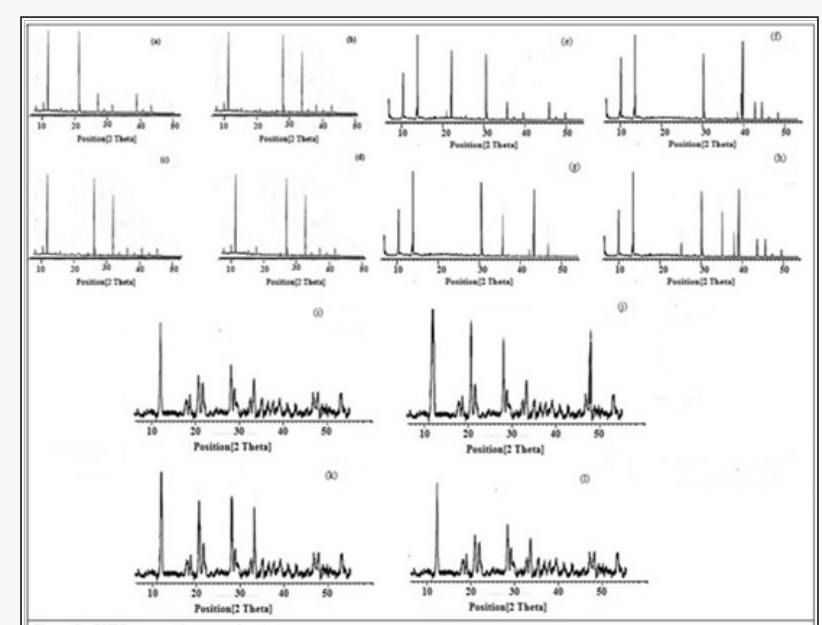

Figure 5: PXRD pattern of compound 3, a) in pure form, b) after terazosine loading, c) after telmisartan loading, d)after glimpiride loading,

PXRD pattern of compound 4, e) in pure form, f) after terazosine loading, g) after telmisartan loading, h) after glimpiride loading,

PXRD pattern of compound 5, i) in pure form, j) after terazosine loading, k) after telmisartan loading, l) after glimpiride loading.

TGA plot of terazosine adsorbed compound 5 (Figure $4 \mathrm{~b}$ ) shows a weight loss of $6 \%$ at $40 \mathrm{oC}$, due to the loss of drug molecules. Second loss of mass at $180 \mathrm{oC}$ is observed and this $4 \%$ weight loss is due to the loss of water molecules. At $280 \mathrm{oC}$ the framework starts to decompose and end up to $580 \mathrm{oC}$ with a final weight loss of $58 \%$. TGA plot of telmisartan adsorbed compound 5 (Figure 4c) shows a weight loss of $4 \%$ at $40^{\circ} \mathrm{C}$ which is due to the loss of telmisartan contents. Another weight loss of $4 \%$ at $150{ }^{\circ} \mathrm{C}$ shows the loss of water molecule. Then the framework starts to decompose at $250^{\circ} \mathrm{C}$ which continues till $550^{\circ} \mathrm{C}$ with a maximum weight loss of $59 \%$. TGA plot of glimpiride adsorbed compound 5 (Figure $4 \mathrm{~d}$ ) shows first weight loss of $4 \%$ at $25^{\circ} \mathrm{C}$ which is due to the removal of glimpiride contents. Then up to $120^{\circ} \mathrm{C}$ the framework remains intact and at this temperature framework shows a weight loss of $4 \%$ up to $190^{\circ} \mathrm{C}$ which is due to removal of water molecule. At $280^{\circ} \mathrm{C}$ the decomposition of the framework and ligands starts which continues till $560^{\circ} \mathrm{C}$ with ultimate weight loss of $58 \%$ (Figure 4).

PXRD patterns of compounds 3-5 were recorded as described in Figure 5. Powder XRD pattern of all these newly synthesized materials after drugs adsorption has revealed the permanent crystalline integrity of these compounds as these have retained its crystallinity even after soaking in water for several days (Figure 5). For the sake of estimation of the drugs contents in the synthesized materials, high Performance Liquid Chromatography was performed on a Waters 2695 separation module. All the details of HPLC parameters used for HPLC studies have been given in Table 1 (supporting information). 0.095g/g, 0.083g/g and 0.041g/ gmaterial of terazosine hydrochloride, telmisartan, and glimpiride has been estimated in compound 3 with a maximum time of release of 5,3 and 3 days respectively. $0.200 \mathrm{~g} / \mathrm{g}, 0.160 \mathrm{~g} / \mathrm{g}$ and $0.138 \mathrm{~g} / \mathrm{g}$ material of terazosine, telmisartan and glimpiride were detected in compound 4. These adsorbed amounts of drugs were slowly released from the compound after different time intervals. The maximum time of release of these drugs was estimated at 7, 5 and 3 days respectively.

The adsorbed amounts of drugs terazosine, telmisartan and glimpiride have been estimated as $0.316 \mathrm{~g} /, 0.086 \mathrm{~g} / \mathrm{g}, 0.138 \mathrm{~g} / \mathrm{g}$, $0.094 \mathrm{~g} / \mathrm{g}$ material of compound 5 . Slow release of these drugs from compound 8 was observed through HPLC. The maximum time of release of these drugs from compound was estimated at 9,3 and 3 days respectively. Table 3 gives summative information 
about the drugs adsorption capacities of compounds 3-5. (Figures 6-17) (supporting information) indicate the HPLC peaks for the estimation of adsorbed drugs in the channels of compound 3-5 and its slow release after different time periods (Table 1).

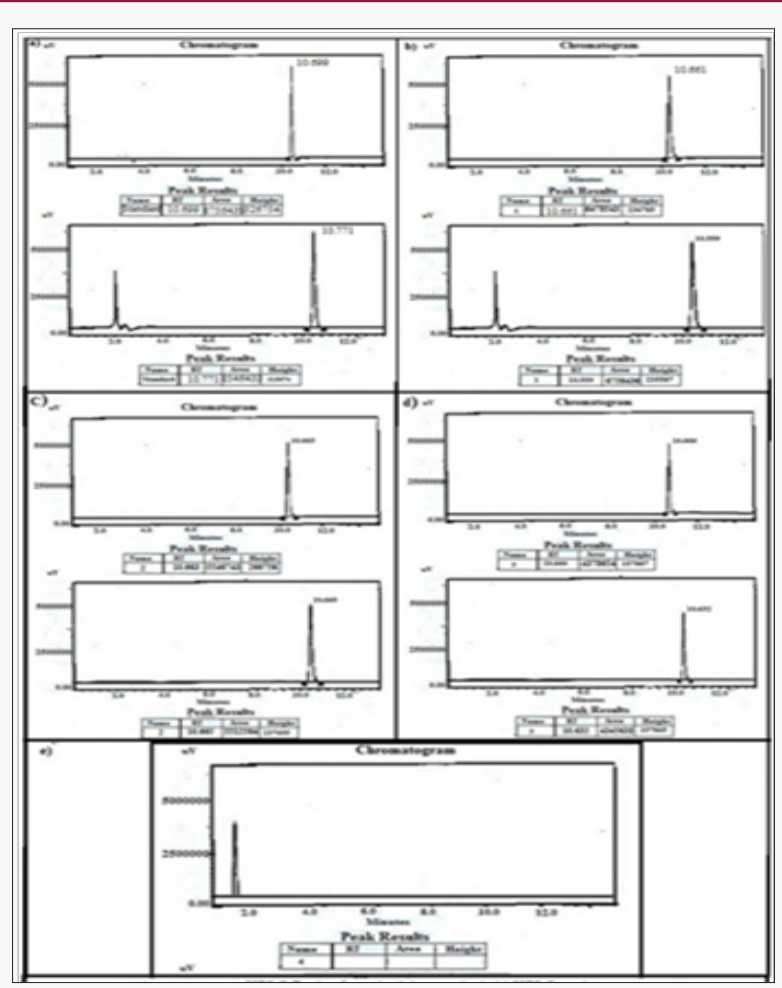

Figure 6: HPLC Peak of standard (Terazosine), b) HPLC peak of samples \#1(aliquot of water containing released drug collected after one day of soaking the drug adsorbed material in distilled water, c) sample \#2 collected after three days, d) Sample \#3: collected after five days, e) Collected after seven days.

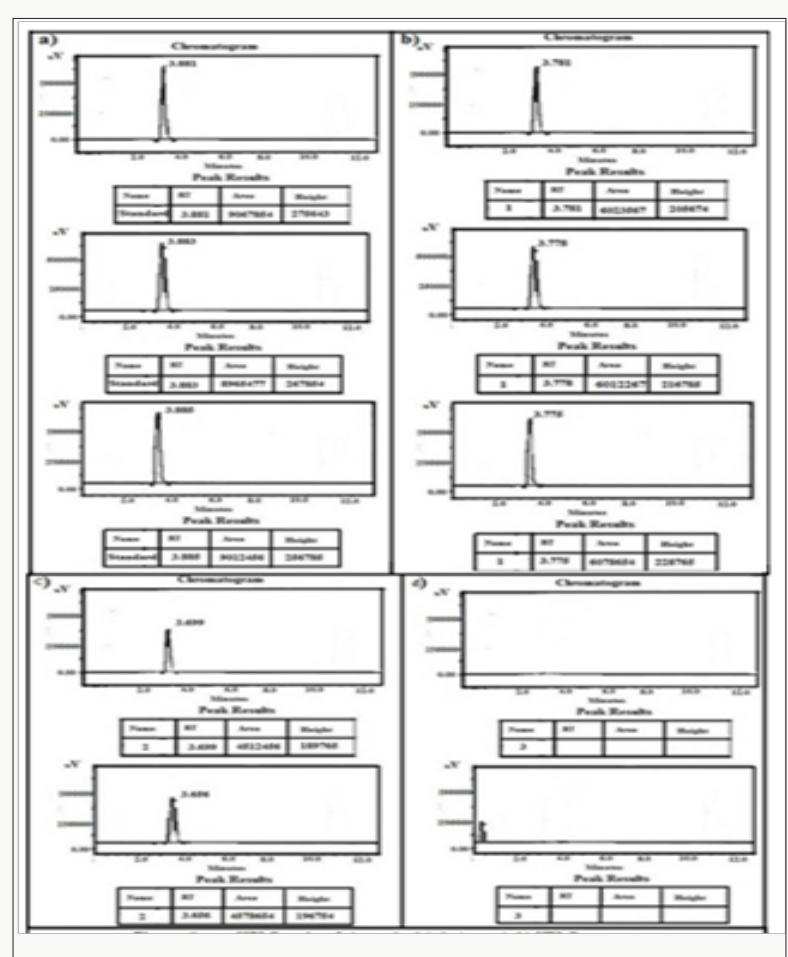

Figure 7: HPLC Peak of standard (Telmisartan), b) HPLC peak of samples \#1(aliquot of water containing released drug collected after one day of soaking the drug adsorbed material in distilled water, c) sample \#2 collected after three days, d) Sample \#3: collected after five days, e) Collected after five days. 


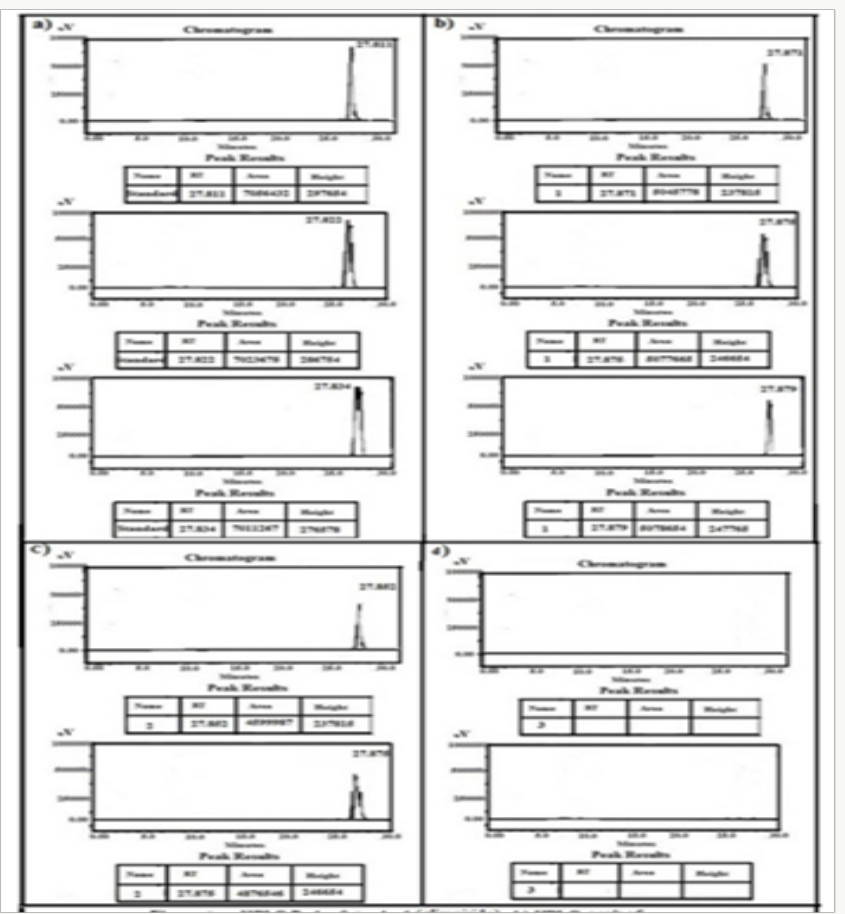

Figure 8: HPLC Peak of standard (glimpiride), b) HPLC peak of samples \#1(aliquot of water containing released drug collected after one day of soaking the drug adsorbed material in distilled water, c) sample \#2 collected after three days, d) Sample \#3: collected after five days, e) Collected after five days.

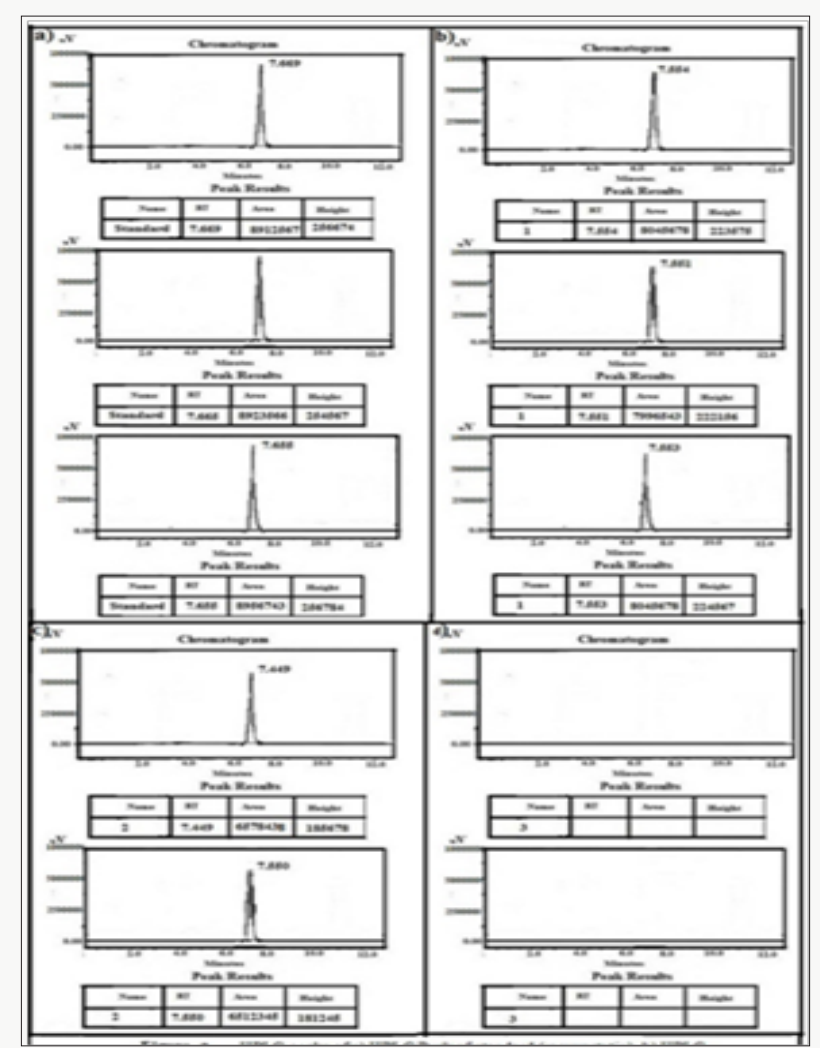

Figure 9: HPLC Peak of a) HPLC Peak of standard (rosuvastatin), b) HPLC peak of samples \#1(aliquot of water containing released drug collected after one day of soaking the drug adsorbed material in distilled water, c) sample \#2 collected after three days, d) Sample \#3: collected after five days, e) Collected after five days. 


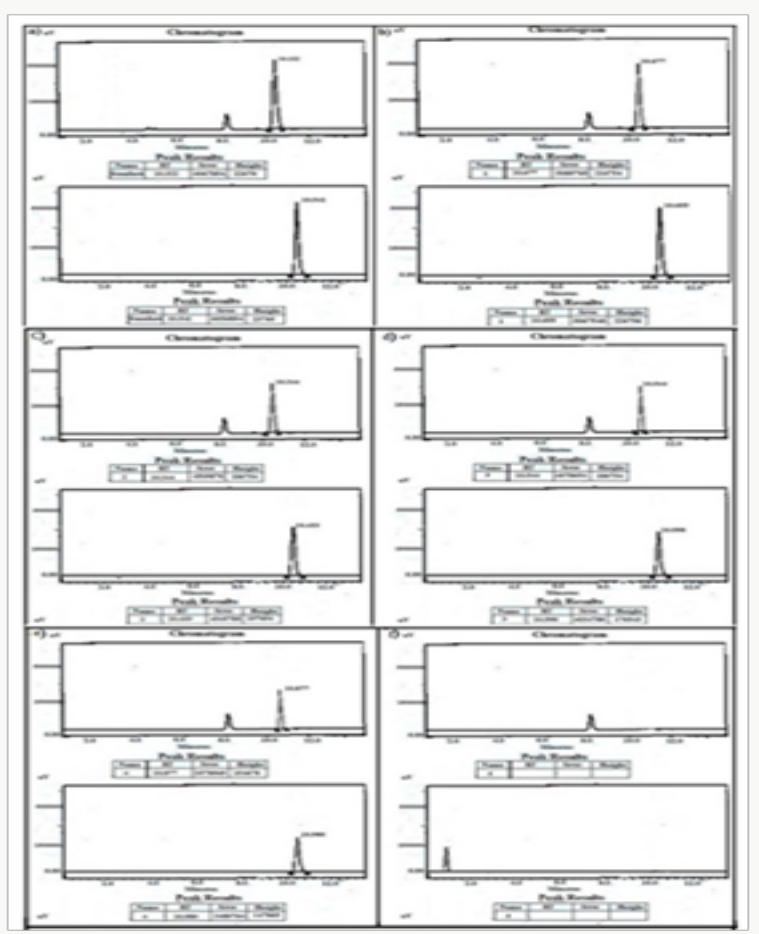

Figure 10: HPLC Peak of a) HPLC Peak of standard (Terazosine), b) HPLC peak of samples \#1(aliquot of water containing released drug collected after one day of soaking the drug adsorbed material in distilled water, c) sample \#2 collected after three days, d) Sample \#3: collected after five days, e) Collected after seven days, f) Samples \#5: collected after nine days.

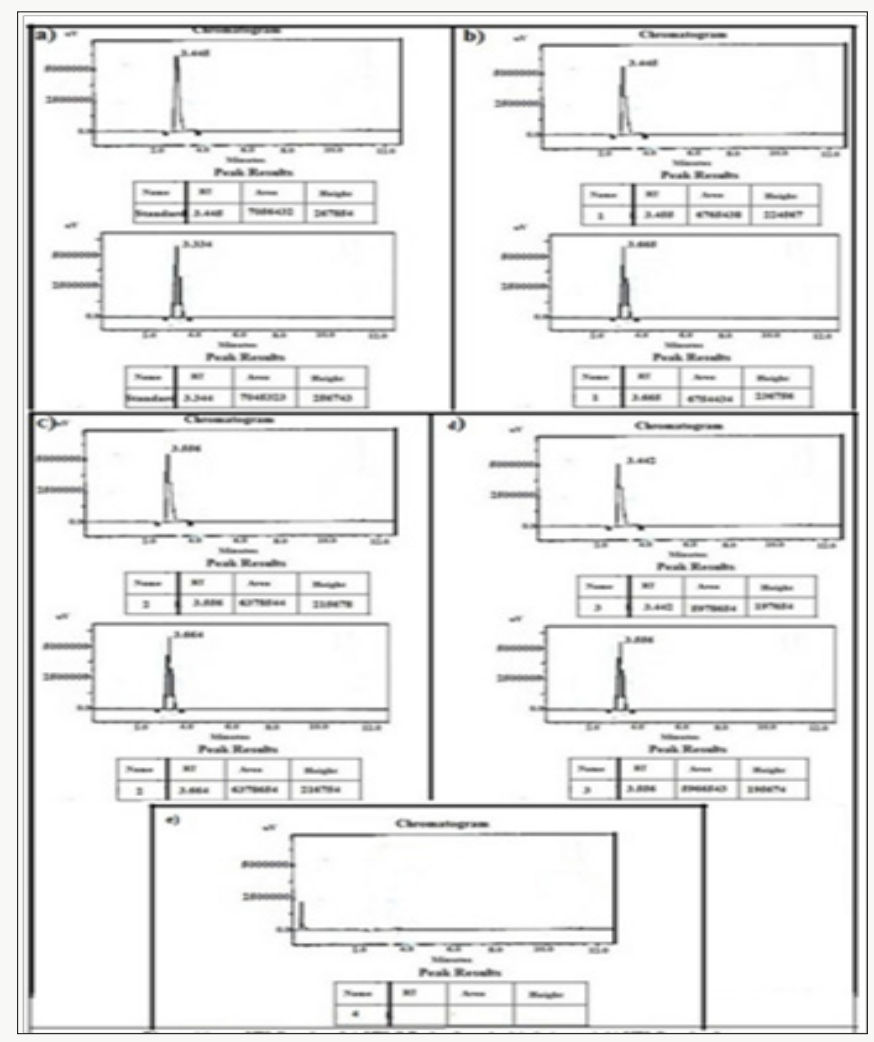

Figure 11: HPLC Peak of a) HPLC Peak of standard (Telmisartan), b) HPLC peak of samples \#1(aliquot of water containing released drug collected after one day of soaking the drug adsorbed material in distilled water, c) sample \#2 collected after three days, d) Sample \#3: collected after five days, e) Collected after seven days, f) Samples \#4: collected after seven days. 


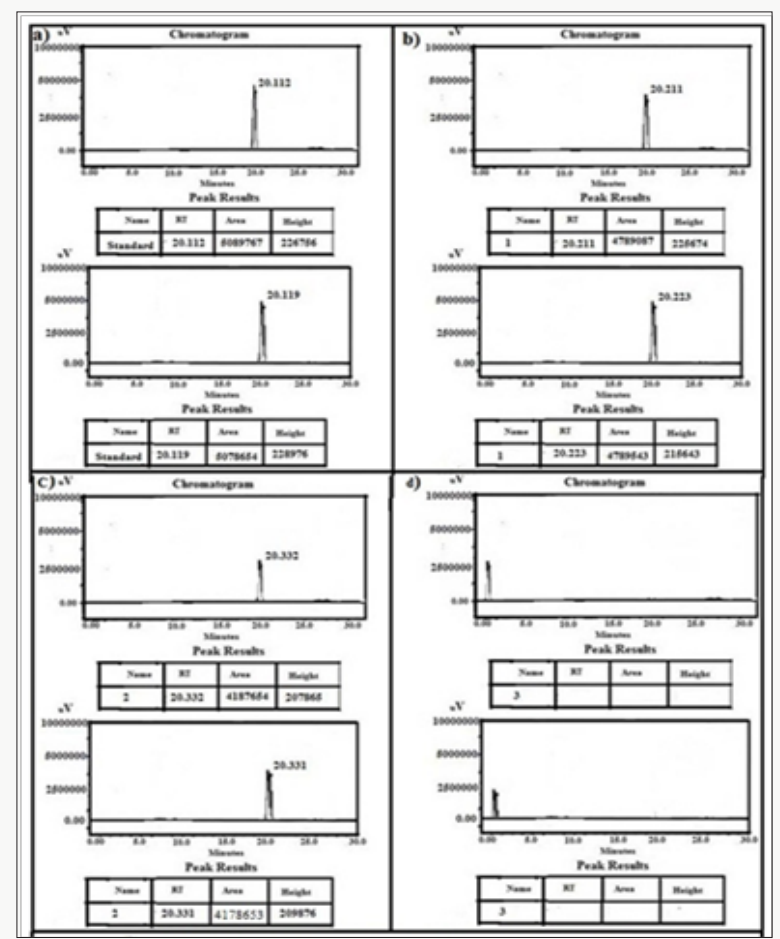

Figure 12: HPLC Peak of a) HPLC Peak of standard (glimpiride), b) HPLC peak of samples \#1(aliquot of water containing released drug collected after one day of soaking the drug adsorbed material in distilled water, c) sample \#2 collected after three days, d) Sample \#3: collected after five days.

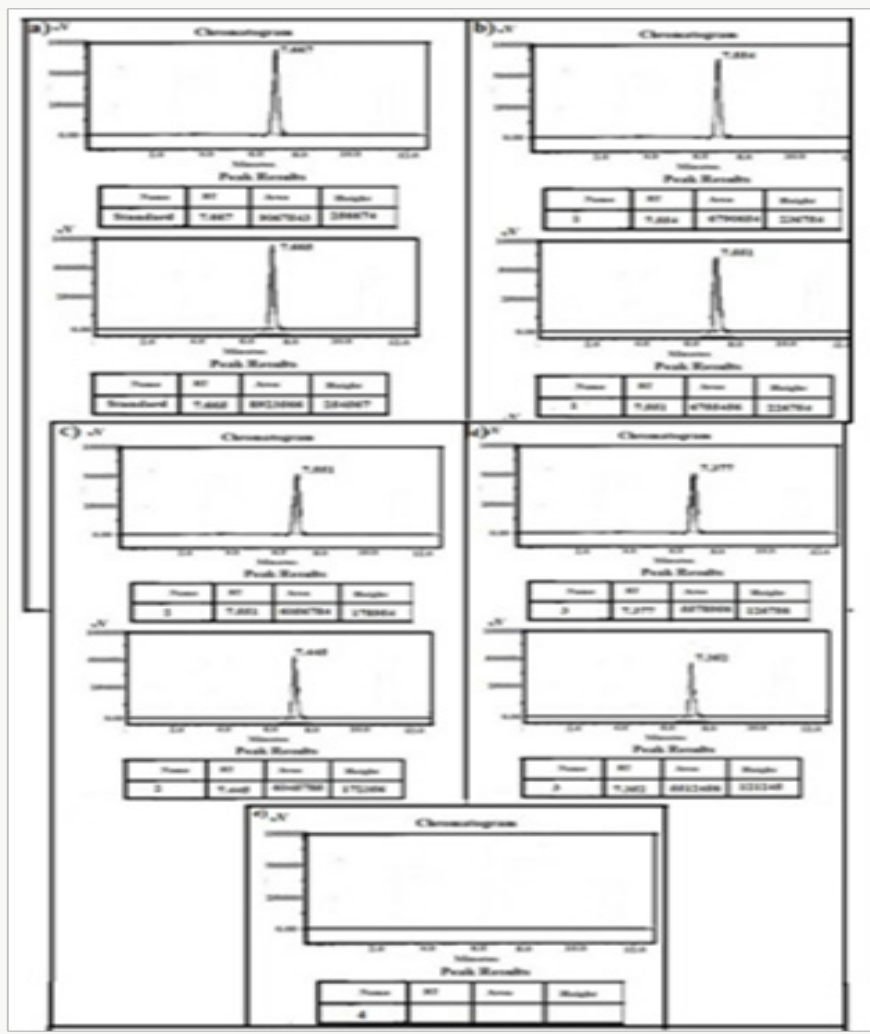

Figure 13: HPLC Peak of a) HPLC Peak of standard (rosuvastatin), b) HPLC peak of samples \#1(aliquot of water containing released drug collected after one day of soaking the drug adsorbed material in distilled water, c) sample \#2 collected after three days, d) Sample \#3: collected after five days, Sample \#4: Collected after seven days. 


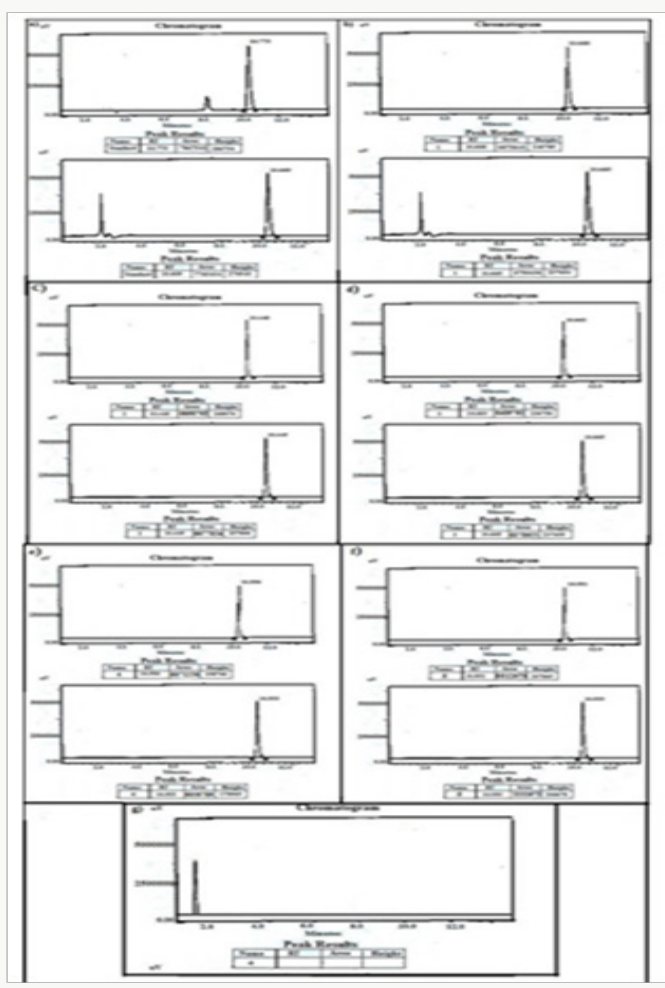

Figure 14: HPLC Peak of a) HPLC Peak of standard (Terazosine), b) HPLC peak of samples \#1(aliquot of water containing released drug collected after one day of soaking the drug adsorbed material in distilled water, c) sample \#2 collected after three days, d) Sample \#3: collected after five days, Sample \#4: Collected after seven days, f) Sample \#5: collected after nine days, g) Sample \#6: collected after eleven days.

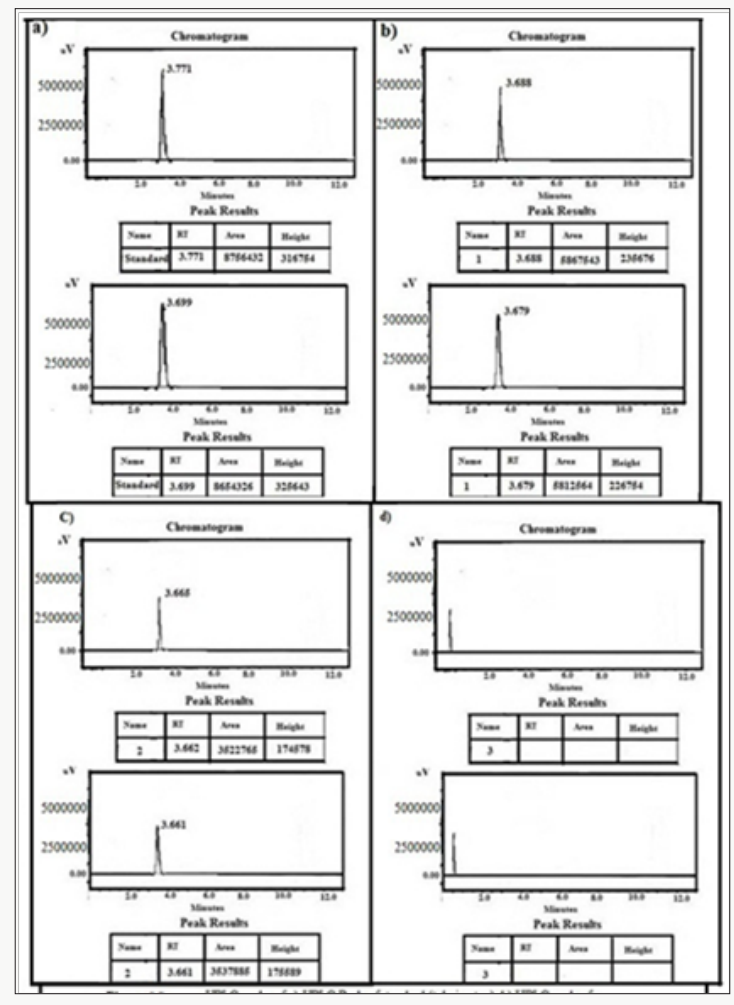

Figure 15: HPLC Peak of a) HPLC Peak of standard (Telmisartan), b) HPLC peak of samples \#1(aliquot of water containing released drug collected after one day of soaking the drug adsorbed material in distilled water, c) sample \#2 collected after three days, d) Sample \#3: collected after five days. 


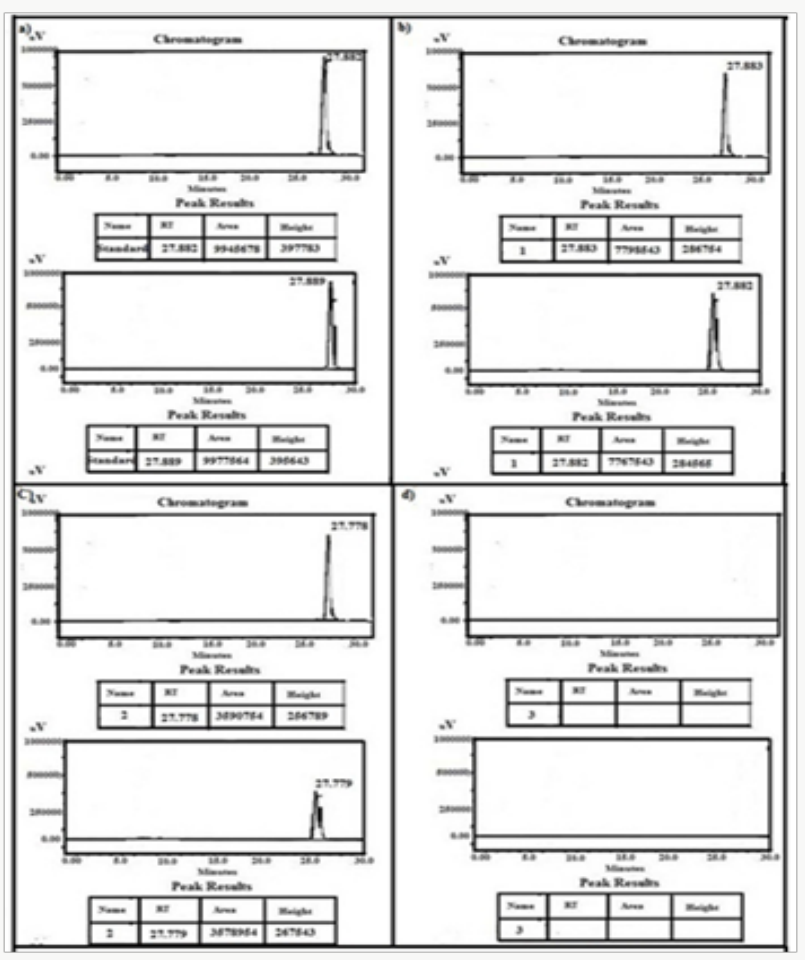

Figure 16: HPLC Peak of a) HPLC Peak of standard (glimpiride), b) HPLC peak of samples \#1(aliquot of water containing released drug collected after one day of soaking the drug adsorbed material in distilled water, c) sample \#2 collected after three days, d) Sample \#3: collected after five days.

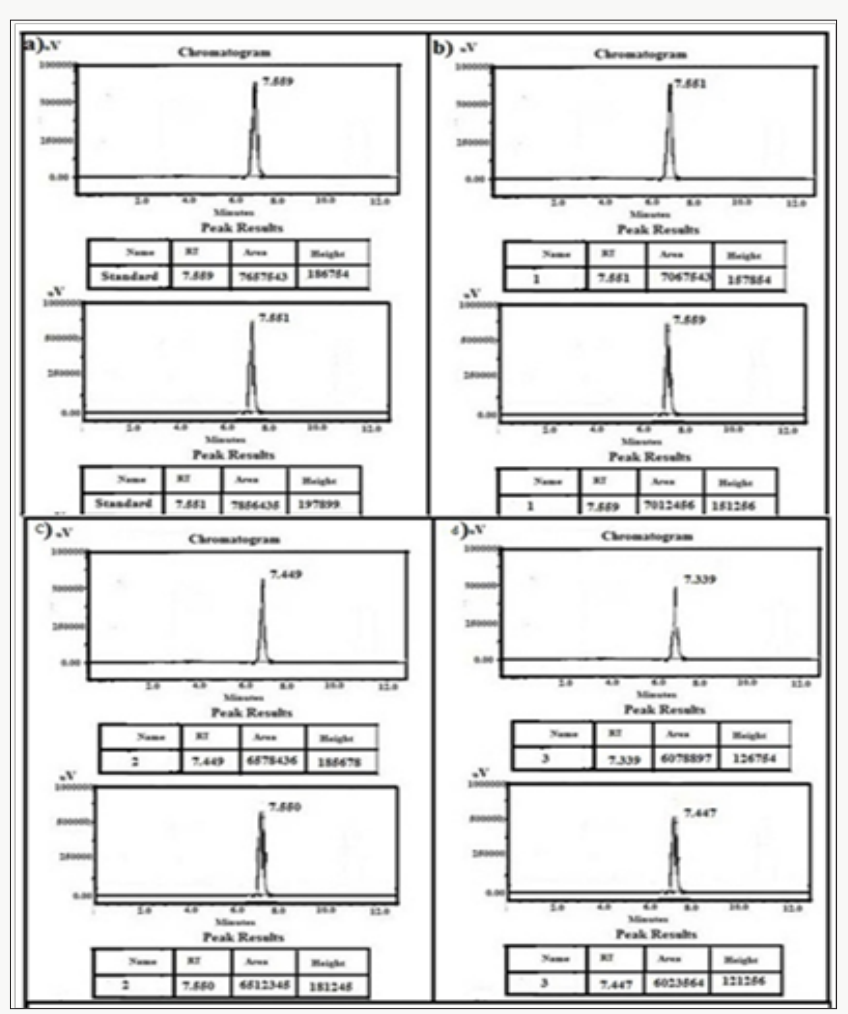

Figure 17: HPLC Peak of a) HPLC Peak of standard (rosuvastatin), b) HPLC peak of samples \#1(aliquot of water containing released drug collected after one day of soaking the drug adsorbed material in distilled water, c) sample \#2 collected after three days, d) Sample \#3: collected after five days, e) Sample \#4: collected after seven days. 
Table 1: A comparison of drugs storage capacities of compounds 3-5.

\begin{tabular}{|c|c|c|c|c|c|}
\hline $\begin{array}{c}\text { Synthesized porous } \\
\text { material }\end{array}$ & $\begin{array}{l}\text { Specific surface areas } \\
\text { (m2g-1) }\end{array}$ & Pore size (A) & $\begin{array}{l}\text { Name of drug } \\
\text { absorbed in } \\
\text { materials }\end{array}$ & $\begin{array}{l}\text { Drug loading } \\
\text { capacity }(\%)\end{array}$ & $\begin{array}{l}\text { Time of released } \\
\text { (days) }\end{array}$ \\
\hline \multirow{3}{*}{ Compound 3} & \multirow{3}{*}{2800} & \multirow{3}{*}{10} & $\begin{array}{c}\text { Terazosine } \\
\text { hydrochloride }\end{array}$ & 0.095 & 5 \\
\hline & & & Telmisartan & 0.083 & 3 \\
\hline & & & Glimpiride & 0.041 & 3 \\
\hline \multirow{3}{*}{ Compound 4} & \multirow{3}{*}{3000} & \multirow{3}{*}{10} & $\begin{array}{c}\text { Terazosine } \\
\text { hydrochloride }\end{array}$ & 0.200 & 7 \\
\hline & & & Telmisartan & 0.160 & 5 \\
\hline & & & Glimpiride & 0.138 & 3 \\
\hline \multirow{3}{*}{ Compound 5} & \multirow{3}{*}{2900} & \multirow{3}{*}{10} & $\begin{array}{c}\text { Terazosine } \\
\text { hydrochloride }\end{array}$ & 0.316 & 9 \\
\hline & & & Telmisartan & 0.086 & 3 \\
\hline & & & Glimpiride & 0.138 & 3 \\
\hline
\end{tabular}

\section{Conclusion}

In conclusion the present work directs towards the use of some nanosized bio-MOFs which were aimed to know their potential for in vitro drugs adsorption. This work elaborates the more potential use of nanoscale bio-MOFs for drugs storage and its release in comparison to previously reported carrier materials. In medical grounds the successful in vitro drug adsorption experiments on these nanosized materials may lead to their usage for in vivo drugs adsorption as well.

\section{References}

1. B Moulton, MJ Zaworotko (2001) from molecules to crystal engineering: supramolecular isomerism and polymorphism in network solids. Chemical Reviews 101(6): 1629-1658.

2. JLC Rowsell, OM Yaghi (2005) Strategies for hydrogen storage in metal-organic frameworks. Angewandte Chemie 44(30): 4670-4679.

3. S Kitagawa, R Kitaura, SI Noro (2004) Functional porous coordination polymers. Angewandte Chemie 43(18): 2334-2375.

4. WJ Rieter, KML Taylor, W Lin (2007) Surface modification and functionalization of nanoscale metal-organic frameworks for controlled release and luminescence sensing. Journal of the American Chemical Society 129(32): 9852-9853.

5. WJ Rieter, KML Taylor, H An, W Lin, W Lin (2006) Nanoscale metal-organic frameworks as potential multimodal contrast enhancing agents. Journal of the American Chemical Society 128(28): 9024-9025.

6. S Wottki, M Lismont, , A Escudero, B Rungtaweevoranit, WJ Parak (2017) Positioning metal-organic framework nanoparticles within the context of drug delivery-A comparison with mesoporous silica nanoparticles and dendrimers. Biomaterials 123: 172-183.
7. KML Taylor, A Jin, W Lin (2008) Surfactant-assisted synthesis of nanoscale gadolinium metal-organic frameworks for potential multimodal imaging. Angewandte Chemie International Edition 47(40): 7722-7725.

8. KML Taylor, WJ Rieter, W Lin (2008) Manganese-based nanoscale metal-organic frameworks for magnetic resonance imaging. Journal of the American Chemical Society 130(44): 14358-14359.

9. M Lismont, L Dreesen, S Wuttki (2017) Metal-Organic Framework Nanoparticles in Photodynamic Therapy: Current Status and Perspectives. Advanced Functional Materials 27(14): 1606314.

10. C He, D Liu, W Lin (2015) Nanomedicine Applications of Hybrid Nanomaterials Built from Metal-Ligand Coordination Bonds: Nanoscale Metal-Organic Frameworks and Nanoscale Coordination Polymers. Chemical Reviews 115: 11079-11108.

11. R Roder, T Preiß, P Hirschle, B Steinborn, A Zimpel, et al. (2017) Multifunctional Nanoparticles by Coordinative Self-Assembly of His-Tagged Units with Metal-Organic Frameworks. Journal of American Chemical Society 139(6): 2359-2368.

12. T Hidalgo, M Giménez-Marqués, E Bellido, J Avila, MC Asensio, et al. (2017) Chitosan-coated mesoporous MIL-100(Fe) nanoparticles as improved bio-compatible oral nanocarriers. Scientific Reports 7: 43099.

13. OR Evans, W Lin (2002) Crystal engineering of NLO materials based on metal-organic coordination networks. Accounts of Chemical Research 35(7): 511-522.

14. R Anand, F Borghi, F Manoli, I Manet, V Agostoni, et al. (2014) HostGuest Interactions in Fe(III)-Trimesate MOF Nanoparticles Loaded with Doxorubicin. Journal of Physical Chemistry 118(29): 8532-8539.

15. T Sattar, M Athar, M Najamul Haq (2016) Hydrothermal Synthesis, Characterization, and In Vitro Drug Adsorption Studies of Some Nano-BioMOFs. Journal of Nanomaterials. 
(C) (i) This work is licensed under Creative

Submission Link: http://biomedres.us/submit-manuscript.php

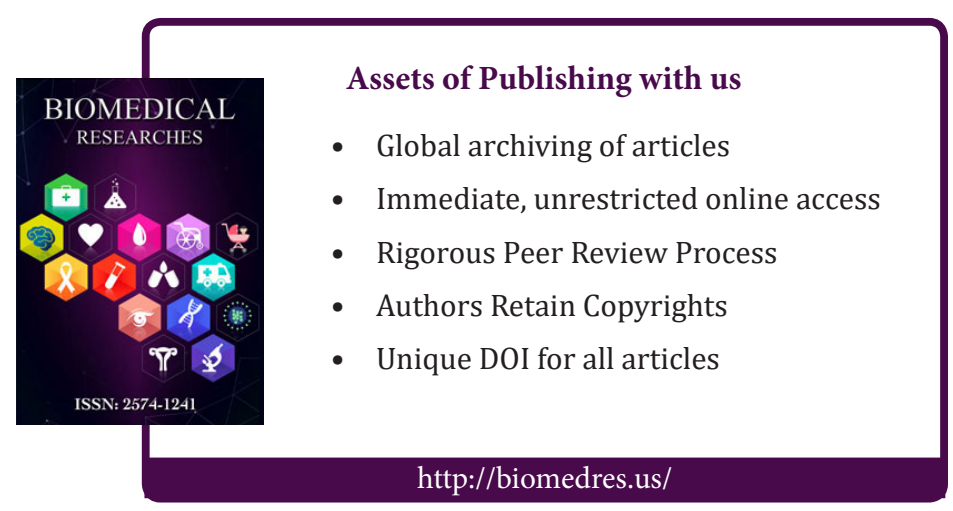

